\title{
HIV prevention for South African youth: which interventions work? A systematic review of current evidence
}

\author{
Abigail Harrison ${ }^{1 *}$, Marie-Louise Newell ${ }^{2,3}$, John Imrie ${ }^{2,4}$, Graeme Hoddinott ${ }^{2}$
}

\begin{abstract}
Background: In South Africa, HIV prevalence among youth aged 15-24 is among the world's highest. Given the urgent need to identify effective HIV prevention approaches, this review assesses the evidence base for youth HIV prevention in South Africa.

Methods: Systematic, analytical review of HIV prevention interventions targeting youth in South Africa since 2000. Critical assessment of interventions in 4 domains: 1) study design and outcomes, 2) intervention design (content, curriculum, theory, adaptation process), 3) thematic focus and HIV causal pathways, 4) intervention delivery (duration, intensity, who, how, where).

Results: Eight youth HIV prevention interventions were included; all were similar in HIV prevention content and objectives, but varied in thematic focus, hypothesised causal pathways, theoretical basis, delivery method, intensity and duration. Interventions were school- (5) or group-based (3), involving in- and out-of-school youth. Primary outcomes included HIV incidence (2), reported sexual risk behavior alone (4), or with alcohol use (2). Interventions led to reductions in STI incidence (1), and reported sexual or alcohol risk behaviours (5), although effect size varied. All but one targeted at least one structural factor associated with HIV infection: gender and sexual coercion (3), alcohol/substance use (2), or economic factors (2). Delivery methods and formats varied, and included teachers (5), peer educators (5), and older mentors (1). School-based interventions experienced frequent implementation challenges.
\end{abstract}

Conclusions: Key recommendations include: address HIV social risk factors, such as gender, poverty and alcohol; target the structural and institutional context; work to change social norms; and engage schools in new ways, including participatory learning.

\section{Background}

With South African youth aged 15-24 experiencing among the highest HIV prevalence in the world [1], the development of effective HIV prevention programmes is a top public health and policy priority [2]. However, in spite of recent calls to increase attention to the high levels of HIV transmission to young women [3], particularly in southern Africa [4], little scientific consensus exists about how best to prevent HIV infection among youth. In countries where HIV prevalence has declined at population level, sexual behaviour change among

\footnotetext{
*Correspondence: abigail_harrison@brown.edu

${ }^{1}$ Brown University, Population Studies and Training Center, and Department of Medicine, Warren Alpert School of Medicine, Providence, RI USA
}

young people has been cited as an important contributing factor [2]. Yet questions remain regarding how to achieve - and maintain - the individual-level behavioural changes needed to reduce HIV incidence.

Comprehensive sexuality education is considered an important means of addressing adolescent risk behaviours [5,6], although little evidence supports its direct impact on biological measures of prevention success, particularly HIV and other sexually transmitted infections (STIs) [5-7]. In sub-Saharan Africa, experience with youth HIV prevention programmes is limited, with evidence regarding effectiveness still emerging. Recent trials of youth HIV prevention interventions have achieved mixed results. Three large community trials of comprehensive approaches to youth HIV prevention, 
involving schools and other key institutions and stakeholders, have failed to significantly reduce HIV incidence in young people, and have shown only modest success in increasing protective behaviours [8-10]. However, two group-based interventions in South Africa have shown promise in reducing reported HIV-related risk behaviours, and in one case, associated biological outcomes [11-13]. Both interventions addressed HIVrelated structural factors, or the social influences underlying HIV risk [14], namely gender-based violence [11-13] and women's poverty [12,13]. Together with limited results of several smaller, school-based interventions, these outcomes have triggered debate about 'which interventions work' [15].

Prior reviews of youth intervention studies in both developed and developing countries [2,5,7,16-19] suggest an important role for school-based interventions in increasing young people's knowledge of sexuality, reproductive health, and HIV prevention, with a majority leading to reductions in reported risk behaviours [5]. Reviews of school-based interventions specific to subSaharan Africa have found greater intervention impact on HIV-related knowledge and attitudes than on reported sexual behaviours [16,17], a finding reinforced by two recent large-scale trials in Tanzania, the Mema kwa Vijana (MkV) Project, and Zimbabwe, the Regai Dzive Shiri (RDS) Project $[9,10]$. These trials impacted knowledge and attitudes (both), self-efficacy (RDS), some aspects of men's sexual behaviour (MkV), and also self-reported pregnancy (RDS). Lessons learned from these trials include the need to change population norms regarding sexual risk behaviours, and to address a broad range of interpersonal, cultural and structural factors underlying HIV risk [20]. Further, strengthening and broadening existing approaches, like school-based programmes, and adopting new approaches to reduce youth HIV incidence in sub-Saharan Africa are important $[16,17,20]$.

To understand current evidence for youth HIV prevention in sub-Saharan Africa, and to answer the question 'which interventions work, and why', reviews of intervention content and characteristics of successful interventions are needed. While several systematic reviews of HIV prevention interventions have included developing country adolescents [2,5,16-18,21], only three focused on sub-Saharan Africa specifically [16,17,21]. Two reviews included only school-based studies [16,17], while the third reviewed studies with an HIV endpoint [21]. Further, many reviews primarily consider methodological and study design issues [16-19]. Reviews may include few details about interventions, or on secondary and process outcomes that indicate pathways to intervention outcomes. Lastly, since systematic reviews are often based on published studies, recently-completed studies may be excluded, although these may offer relevant lessons learned, helping to identify promising approaches.

The debate about 'which interventions work - and why' has been renewed following recent trial results. In South Africa, the continued severity of the HIV epidemic has lent new urgency to these questions - and their answers [22]. The development of interventions specific to the South African context is an urgent research priority [23], and may support intervention approaches for sub-Saharan Africa more broadly. To inform the development of an evidence-based, state-ofthe-art approach to youth HIV prevention in South Africa, we undertook a review of ongoing or recently completed intervention studies, with the aim of systematically assessing characteristics of rigourously designed youth HIV prevention interventions, to better understand how they work, and why.

\section{Methods}

This review addresses study design, intervention design, including content and theoretical basis, thematic focus and HIV causal pathways, and intervention delivery and implementation, among youth aged 12-24 years in South Africa. The review methodology was adapted from other reviews of adolescent HIV prevention studies, to ensure rigorous and systematic assessment of the strength of evidence on intervention effectiveness $[2,5,18,19,23]$.

\section{Inclusion Criteria}

'Rigorously designed' programs were defined as those focused specifically on preventing or reducing HIVrelated risk behaviours in young people, or their determinants, or incidence of HIV or other sexually transmitted infections. Selection criteria included: 1) intervention conducted in South Africa, in 2000 or after (since interventions prior to that date focused primarily on increasing knowledge and changing attitudes, and have been included in other reviews); 2) behavioural intervention focused on youth, using a broad definition of 12-24 years of age; 3 ) experimental design including a control or comparison condition; 4) biological or behavioural outcome, or both; and 5) available information on intervention structure (eg., curriculum, group vs individual format, number of sessions, implementation/ delivery personnel), content (eg., major themes and topics, information provided, targeted risk/protective factors), and theoretical framework. To consider the most up-to-date evidence, we included three types of studies: 1) completed interventions with published findings; 2) completed interventions with unpublished evaluations; and 3) promising approaches, which were ongoing interventions that met other criteria, with 
sufficient data available to assess the intervention's content and likely impact. The result is a systematically conducted review of current youth HIV prevention interventions in South Africa.

The search strategy included computerised searches of MEDLINE, Social Sciences Index, and the NIH search engine CRISP; abstracts from the 2004, 2006 and 2008 International AIDS conferences; examination of alreadypublished systematic reviews [16,17], and expert consultation, creating a 'snowball' effect when other appropriate interventions were identified. Search terms included "schools, HIV prevention, South Africa" and "youth, HIV/AIDS, South Africa." We distinguished between 'programmes' and 'interventions', and excluded national programmes, such as LoveLife or Soul City [24,25], as well as programmes designed to support the national LifeSkills programme and their evaluations [26-28], since these tended to be larger, with a geographically broader target population, and often had multiple components that rendered the evaluation of impact in a specific population more difficult. Instead, the review included interventions that were curriculum-based, or followed a similar structured protocol, aimed at effecting behavioural changes leading to a decrease in HIV incidence or related risk behaviours among South African youth. Some print media or health communication evaluations not explicitly designed to measure behaviour change were also excluded [29].

\section{Selection of Studies}

Nine intervention studies were initially identified [11,13,30-36]. After further review, one of the nine although a promising model based on a community participatory approach - was excluded as it was a family intervention targeting parents, caregivers and pre-adolescents, and did not measure impact in youth aged 1224 [30]. One of the eight selected was not formally a 'youth' intervention, but included an evaluation of the program's impact on young women and was thus included, although results need to be interpreted cautiously as the study was not designed with sufficient power to measure outcomes in this sub-group [13]. Following identification of the studies, informal interviews were conducted with at least one researcher from each selected intervention, and requests made for copies of project materials. The process was further informed by an expert scientific workshop convened to discuss stateof-the-art issues related to schools and youth HIV prevention research.

\section{Analytical Process}

Information from these sources, as well as published reports, was used to prepare a description of each intervention, highlighting the substantive focus and hypothesised pathways to achieving the study's primary outcome(s). The main analytical categories were specified a priori, based on characteristics of interventions demonstrated to be successful in diverse settings $[5,16,17]$. These included: 1) process of intervention development, including formative research; 2) cultural/linguistic adaptation; 3) use of social/behavioural theory; 4 ) how and where the intervention was delivered (eg. classroom, community, after school, extra periods); 5) who delivered the intervention (eg., peer educators, teachers, trained facilitators); 6) selection and reinforcement of key messages; 7) involvement of participants and/or broader community; 8) focus on social context and risk environments, as well as individual risk behaviours; and 9) focus on HIV causal pathways of relevance to South African setting.

\section{Results}

\section{Study Design and Outcomes}

Eight interventions were included in the review. Stepping Stones, which reduced incidence of HSV-2, was one of two studies to measure biological outcomes, and the only one to report a significant impact [11]. Five of eight interventions demonstrated a significant improvement in reported HIV-related risk behaviours $[11,13,32,33,35]$, including condom use [33], HIV testing $[13,35]$, heavy drinking [11] or alcohol use during sexual activity [32], and intimate partner violence $[11,13]$. Two other interventions resulted in maintenance of reported risk behaviours in the intervention group, with increased reports of sexual or alcohol risk behaviour in the comparison group [34,36], while one intervention showed no effect at all [31] (Table 1). Although all studies employed a control or comparison group, experimental designs varied widely, with some having non-randomised selection, multiple versus single assessments, sample sizes ranging from several hundred to several thousand participants, differences in length of follow-up period, active versus passive controls and cluster versus individually-randomised designs (Table 1). These differences contributed to a range of effect sizes, making comparison of intervention outcomes and impact difficult (Table 2). Further, assessment methods differed: surveys were conducted using electronic data capture via PDA (personal digital assistant) [31], self-administered classroom surveys using pen and paper $[32,33,35,36]$, or individual assessment by interviewers $[11,13,34]$. All interventions conducted 'process' evaluations - generally qualitative to monitor intervention implementation (Table 1), and several studies had additional published reports on details of study design [37-40] or qualitative assessments [41,42]. 
Table 1 Study Design for Eight Youth HIV Prevention Interventions in Systematic Review

\begin{tabular}{|c|c|c|c|c|c|}
\hline $\begin{array}{l}\text { Project } \\
\text { Description }\end{array}$ & Objective & $\begin{array}{l}\text { Target Population/ } \\
\text { Age }\end{array}$ & $\begin{array}{l}\text { Experimental Design \& } \\
\text { Sample Size }\end{array}$ & $\begin{array}{l}\text { Control or Comparison } \\
\text { Condition }\end{array}$ & $\begin{array}{l}\text { Duration of } \\
\text { Follow-up }\end{array}$ \\
\hline $\begin{array}{l}\text { HAPS [32] } \\
\text { [HIV/AIDS } \\
\text { Prevention } \\
\text { Study] } \\
\text { KwaZulu/Natal }\end{array}$ & $\begin{array}{l}\text { Reduce sexual and alcohol risk- } \\
\text { taking behaviors }\end{array}$ & $\begin{array}{l}\text { Secondary School } \\
\text { students, Grade 9; } \\
\text { Ages: } 14-16 \text { years }\end{array}$ & $\begin{array}{l}\text { Random assignment at } \\
\text { school level; } 3 \text { intervention, } \\
2 \text { comparison schools } \\
\mathrm{N}=325 \mathrm{l} ; \mathrm{N}=336 \mathrm{C} ; \\
\text { pre-post survey }\end{array}$ & $\begin{array}{l}\text { Standard life skills/life } \\
\text { orientation curriculum }\end{array}$ & $\begin{array}{l}\text { Follow up } \\
\text { survey: } 2 \\
\text { months post- } \\
\text { intervention }\end{array}$ \\
\hline $\begin{array}{l}\text { HealthWise [34] } \\
\text { CapeTown }\end{array}$ & $\begin{array}{l}\text { Reduce STI/HIV transmission, } \\
\text { drug/alcohol abuse and } \\
\text { increase positive use of leisure } \\
\text { time }\end{array}$ & $\begin{array}{l}\text { Secondary school } \\
\text { students, Grades 8-9 } \\
\text { Ages 12-14 in urban } \\
\text { township setting; }\end{array}$ & $\begin{array}{l}\text { Pre-and post-intervention } \\
\text { surveys; in } 38^{\text {th }} \text { grade } \\
\text { cohorts; } \\
4 \text { intervention, } 5 \text { control } \\
\text { schools randomly assigned; } \\
\mathrm{N}=901 \mathrm{l} ; \mathrm{N}=1275 \mathrm{C}\end{array}$ & $\begin{array}{l}\text { Standard life skills/life } \\
\text { orientation curriculum }\end{array}$ & $\begin{array}{l}5 \text { waves of data } \\
\text { collection for } \\
\text { each cohort } \\
\text { over } 1.5 \text { years }\end{array}$ \\
\hline $\begin{array}{l}\text { Mpondombili } \\
\text { Project [33] } \\
\text { KwaZulu/Natal }\end{array}$ & $\begin{array}{l}\text { Promote safer sex behaviors, } \\
\text { with emphasis on dual } \\
\text { protection, sexual risk-reduction, } \\
\text { and promotion of positive } \\
\text { gender role norms }\end{array}$ & $\begin{array}{l}\text { Secondary School } \\
\text { students: Grades 8- } \\
10 \text { in rural secondary } \\
\text { schools }\end{array}$ & $\begin{array}{l}\text { Baseline and follow up } \\
\text { surveys in } 2 \text { intervention and } \\
2 \text { comparison schools, not } \\
\text { randomized; } N=442 \mathrm{l} ; \\
\mathrm{N}=541 \mathrm{C}\end{array}$ & $\begin{array}{l}\text { Standard life skills/life } \\
\text { orientation curriculum; } \\
\text { comparison schools } \\
\text { received shortened } \\
\text { version of curriculum } \\
\text { (delayed) }\end{array}$ & $\begin{array}{l}\text { Follow up } \\
\text { survey: } 5 \\
\text { months post- } \\
\text { intervention }\end{array}$ \\
\hline $\begin{array}{l}\text { Adolescent } \\
\text { Livelihoods[35] } \\
\text { KwaZulu/Natal }\end{array}$ & $\begin{array}{l}\text { Reduce HIV risks and social } \\
\text { vulnerabilities, increase access } \\
\text { to 'safe spaces' and life skills }\end{array}$ & $\begin{array}{l}\text { Urban township; Out } \\
\text { of school youth } \\
\text { aged 16-24; in- } \\
\text { school youth aged } \\
14-20\end{array}$ & $\begin{array}{l}\text { Quasi-experimental; group } \\
\text { assignment }\end{array}$ & & 2 years \\
\hline $\begin{array}{l}\text { SATZ } \\
{[31,37,51,52]} \\
\text { CapeTown and } \\
\text { Northern } \\
\text { Province }\end{array}$ & $\begin{array}{l}\text { To develop, implement and } \\
\text { evaluate a school-based health } \\
\text { education program aimed at } \\
\text { promotion of correct, } \\
\text { consistent condom use and } \\
\text { delay in sexual debut }\end{array}$ & $\begin{array}{l}\text { School students in } \\
\text { urban township and } \\
\text { rural area; ages 12-14 } \\
\text { in grade } 8\end{array}$ & $\begin{array}{l}1 \text { pre- and } 2 \text { post-test } \\
\text { assessments within quasi- } \\
\text { experimental design; } 13 \\
\text { intervention and } 13 \text { control } \\
\text { schools; not randomized. } \\
\mathrm{N}=3625\end{array}$ & $\begin{array}{l}\text { Comparison schools } \\
\text { received delayed } \\
\text { intervention }\end{array}$ & 1 year \\
\hline $\begin{array}{l}\text { Stepping } \\
\text { Stones }[11,38] \\
\text { Eastern Cape }\end{array}$ & $\begin{array}{l}\text { Promote sexual and } \\
\text { reproductive health via HIV } \\
\text { prevention and reduction in } \\
\text { sexual coercion and intimate } \\
\text { partner violence }\end{array}$ & $\begin{array}{l}\text { Semi-urban } \\
\text { township; older } \\
\text { adolescents and } \\
\text { young adults aged } \\
\text { 18-24; in- and out- } \\
\text { of-school youth }\end{array}$ & $\begin{array}{l}\text { Cluster RCT; matched control } \\
\text { group; } 35 \text { I clusters; } 35 \mathrm{C} \\
\text { clusters. Sample size: } 2770 \\
\mathrm{~N}=1140 \text { I; } \mathrm{N}=1081 \mathrm{C}\end{array}$ & $\begin{array}{l}\text { Single session on HIV, } \\
\text { condoms, safe sexual } \\
\text { behaviors }\end{array}$ & $\begin{array}{l}2 \text { years post- } \\
\text { intervention } \\
\text { with } 2 \\
\text { assessments, at } \\
12 \text { months and } \\
24 \text { months }\end{array}$ \\
\hline $\begin{array}{l}\text { Tshwane Peer } \\
\text { Education and } \\
\text { Support } \\
\text { Programme[36] } \\
\text { Tshwane } \\
\text { (Pretoria), } \\
\text { Gauteng }\end{array}$ & $\begin{array}{l}\text { Promote accurate information } \\
\text { about HIV/AIDS, address peer } \\
\text { norms, establish psychosocial } \\
\text { support }\end{array}$ & $\begin{array}{l}\text { High school students } \\
\text { ages } 13-20 \text {, in semi- } \\
\text { rural secondary } \\
\text { schools }\end{array}$ & $\begin{array}{l}13 \text { intervention and } 4 \\
\text { control schools; not } \\
\text { randomized. Pre-post survey } \\
\text { of one selected class in each } \\
\text { school. } \\
\mathrm{N}=1572 \mathrm{l} ; \mathrm{N}=596 \mathrm{C}\end{array}$ & $\begin{array}{l}\text { Ongoing Life Orientation } \\
\text { or other HIV prevention } \\
\text { activities }\end{array}$ & 18 months \\
\hline $\begin{array}{l}\text { IMAGE - } \\
\text { Intervention } \\
\text { with } \\
\text { Microfinance } \\
\text { for AIDS and } \\
\text { Gender Equity } \\
{[12,13]}\end{array}$ & $\begin{array}{l}\text { To evaluate effects of } \\
\text { combined microfinance and } \\
\text { training intervention on HIV risk } \\
\text { behavior }\end{array}$ & $\begin{array}{l}\text { Of } 3 \text { evaluation } \\
\text { cohorts, one cohort } \\
\text { of } 14-35 \text { year old } \\
\text { women }\end{array}$ & $\begin{array}{l}\text { Cluster RCT; } 8 \text { pair-matched } \\
\text { villages. } \mathrm{N}=130(\mathrm{I}) ; \mathrm{N}=132 \\
\text { (C) }\end{array}$ & $\begin{array}{l}\text { Villages randomised to } \\
\text { control received } \\
\text { standard of care; } \\
\text { available sexual health } \\
\text { info; no microfinance }\end{array}$ & 2 years \\
\hline
\end{tabular}

\section{Intervention Design}

Five interventions were school-based [31-34,36], and employed a standard classroom approach to intervention delivery, while the remaining three were group-based $[11,13,35]$ and delivered in community venues or schools after-hours (Additional file 1: Table S3). All eight interventions included broadly similar information about HIV transmission and prevention, although relative emphasis on condoms and abstinence/delay of sexual activity, and related topics such as sexual negotiation, gender issues and sexual coercion, self esteem and interpersonal communication, and drugs and alcohol varied. Several interventions addressed pregnancy and contraception, while a few emphasized partner reduction and safe partnerships, and HIV testing $[11,13,31,33]$. Two interventions strongly emphasized broader life skills, including numeracy [35], or training in economic support $[13,35]$. With the exception of the IMAGE and HAPS projects [13,32], all interventions were single-component (Additional file 1, Table S3). 
Table 2 Study Results for Eight Youth HIV Prevention Interventions included in Systematic Review ${ }^{1}$

\begin{tabular}{|c|c|c|c|c|}
\hline Project Name and Location & $\begin{array}{l}\text { Impact of } \\
\text { Intervention on: } \\
\text { Knowledge, } \\
\text { Attitudes, } \\
\text { Perceptions, Social } \\
\text { Norms }\end{array}$ & $\begin{array}{l}\text { Behavioral skills and } \\
\text { intentions for risk } \\
\text { reduction } \\
\text { (communication, } \\
\text { negotiation, self- } \\
\text { efficacy) }\end{array}$ & $\begin{array}{l}\text { Sexual and other HIV risk } \\
\text { behaviours }\end{array}$ & $\begin{array}{l}\text { Clinical and } \\
\text { biological } \\
\text { outcomes and/or } \\
\text { Structural and } \\
\text { Community Effects }\end{array}$ \\
\hline $\begin{array}{l}\text { HAPS [32] } \\
\text { [HIV/AIDS Prevention Study] } \\
\text { KwaZulu/Natal } \\
\text { NB: Study results presented as scores } \\
\text { from scaled measures; analyzed using } \\
\text { ANOVA for each group, and showing } \\
\text { net intervention effect for difference } \\
\text { between intervention (I) and } \\
\text { comparison (C) groups }\end{array}$ & $\begin{array}{l}\text { Positive attitudes to } \\
\text { condom use } \\
0.171 \text { vs } 0.08 C \text {, interv } \\
\text { effect: } 0.09 \\
\text { Positive attitudes } \\
\text { toward alcohol } \\
-0.011 \text { vs } 0.16 C \text {, interv } \\
\text { effect: }-0.17 \\
\text { Negative attitudes } \\
\text { toward alcohol } \\
-0.15 \text { I vs }-0.08 C \text {, } \\
\text { interv effect: }-0.07\end{array}$ & $\begin{array}{l}\text { Self efficacy for sex } \\
\text { refusal } \\
0.081 \text { vs- } 0.16 C \text {, } \\
\text { intervention effect: } \\
0.24 \\
\text { Self efficacy for } \\
\text { condom use } \\
-0.081 \text { vs - } 0.06 C \text {, } \\
\text { intervention effect: } \\
-0.02 \\
\text { Self-efficacy for alcohol } \\
\text { refusal } \\
0.17 \text { vs } 0.44 C \text {, } \\
\text { intervention effect: } \\
-0.27 \\
\text { Condom use } \\
\text { intentions: } \\
0.181 \text { vs -0.01C, } \\
\text { intervention effect: } \\
0.19 \\
\text { Intentions to have sex } \\
\text { - next } 3 \text { months: } 0.001 \\
\text { vs } 0.06 C \text {, intervention } \\
\text { effect: - } 0.06\end{array}$ & $\begin{array}{l}\text { Alcohol Behaviors: } \\
\text { Frequency of alcohol use: last } 14 \\
\text { days } \\
0.231 \text { vs } 0.28 C \text {, intervention effect: } \\
-0.05 \\
\text { Number of drinks last time drinking } \\
0.361 \text { vs }-0.08 C \text {, intervention effect: } \\
0.44 \\
\text { Self or partner drinking at last sex } \\
-0.91 \text { vs } 4.5 C \text {, intervention effect: }-4.4 \\
\text { HIV-related behavior: } \\
\text { Condom use at last sex } \\
4.21 \text { vs } 2.2 C \text {, intervention effect: } 2.0\end{array}$ & Not measured \\
\hline $\begin{array}{l}\text { HealthWise [34] } \\
\text { CapeTown } \\
\text { NB: Assessment of intervention } \\
\text { conducted in } 5 \text { waves of data } \\
\text { collection; unless otherwise specified, } \\
\text { results are presented as comparison } \\
\text { between intervention (I) and } \\
\text { comparison (C) groups at last followup } \\
\text { (wave 5) }\end{array}$ & 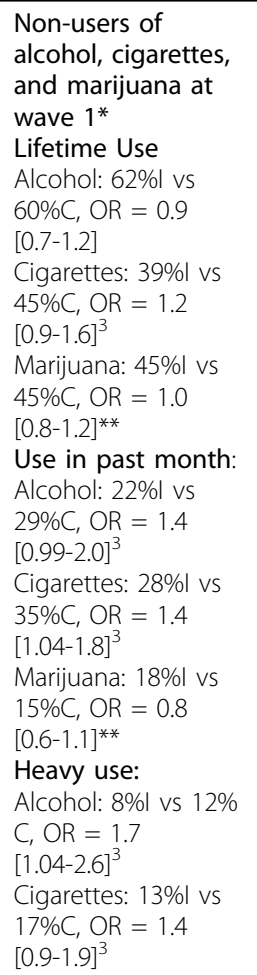 & 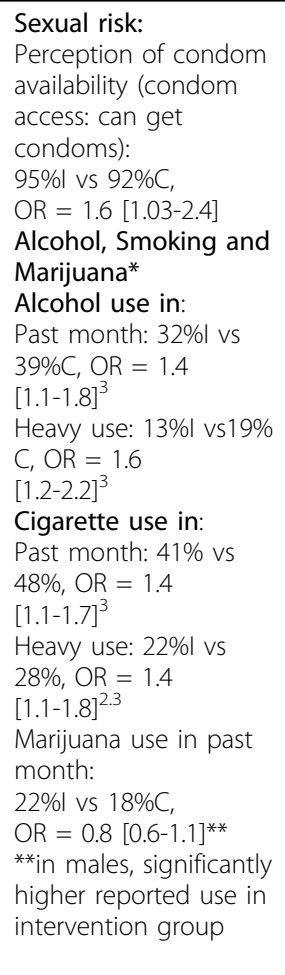 & $\begin{array}{l}\text { Sexual Behavior } \\
\text { Sexually active (lifetime) among } \\
\text { those not sexually experience at } \\
\text { baseline: } \\
22 \% \text { l vs } 21 \% \text { C, OR }=1.0[0.8-1.3] \\
\text { Frequency of: } \\
\text { Sexual activity (past month)***: } \\
-6 \text { I [-19-+6] vs -2C }[-12-+8] \\
\text { Condom use (always)**: } \\
0 \text { I [-12 -+12] vs }+2 C[-9-+12] \\
\text { *Since knowledge and attitudes of } \\
\text { alcohol and other substances were } \\
\text { not reported, these columns report } \\
\text { data on use behaviors in those } \\
\text { experienced (column 2) and not } \\
\text { experienced (column 1) at baseline. } \\
\text { ***reported as 'change in } \\
\text { prevalence' between waves } 4 \text { and } 5 \\
\text { of assessment }\end{array}$ & Not measured \\
\hline
\end{tabular}


Table 2: Study Results for Eight Youth HIV Prevention Interventions included in Systematic Review ${ }^{\mathbf{1}}$ (Continued)

\begin{tabular}{|c|c|c|c|c|}
\hline $\begin{array}{l}\text { Mpondombili } \\
\text { Project [33] KwaZulu/Natal } \\
\text { NB: *denotes significance at } p<0.05 \\
\text { level, based on analysis of data using } \\
\text { regression methods for comparison of } \\
\text { I and C groups at follow up. }\end{array}$ & $\begin{array}{l}\text { General HIV } \\
\text { Knowledge }{ }^{3}: 67.3 \% \text { l } \\
\text { vs } 54.7 C \text {, beta } \\
\text { coefficient: } .26 \\
{[-4.30-+4.35]^{*}} \\
\text { Know where to } \\
\text { access HIV test }{ }^{2}: \\
76.6 \% \text { vs } 62.6 \% C \text {, } \\
\text { beta: } 0.57 \text { [0.08-1.05] } \\
\text { Peer norms for } \\
\text { abstinence (mean } \\
\text { score): } 3.381 \text { vs } 3.67 C \text {, } \\
\text { beta: } 0.28 \\
{[-0.04-0.59]^{*}} \\
\text { Peer norms for girls' } \\
\text { condom use (mean): } \\
2.421 \text { vs } 2.24 C, \text { beta: } \\
-0.07 \text { [-0.27-0.12] } \\
\text { Egalitarian beliefs } \\
\text { about sex refusal: } \\
6.271 \text { vs } 6.07 C, \text { beta: } \\
0.38 \text { [-0.20-0.97] }\end{array}$ & 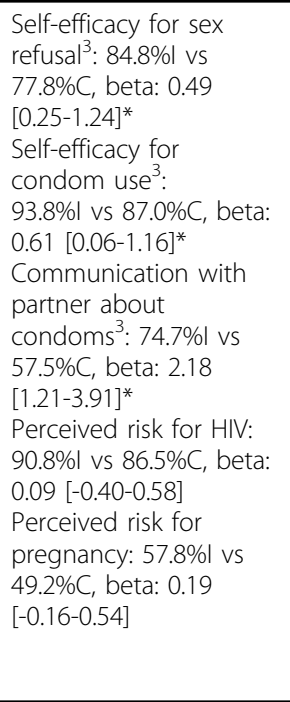 & $\begin{array}{l}\text { Ever condom use }{ }^{3}: 67.3 \% \text { I vs } 41.9 \% \\
\text { C, OR }=2.85[1.62-5.04]^{*} \\
\text { Condom use at last sexual } \\
\text { intercourse }{ }^{2,3}: \\
54.3 \% \text { l vs } 27.9 \% \text { C, OR }=3.21 \\
{[1.76-5.85]^{*}} \\
\text { Ever had sex }: 28.5 \% \text { vs } 22.2 \% \text { C, OR } \\
=1.39[1.02-1.91]^{*}\end{array}$ & Not measured \\
\hline $\begin{array}{l}\text { Adolescent Livelihoods [35] } \\
\text { KwaZulu/Natal } \\
\text { NB: Preliminary findings. No measures } \\
\text { of significance provided. }\end{array}$ & $\begin{array}{l}\text { Indicators of } \\
\text { financial well-being } \\
\text { Has savings: } \\
\text { M: } 34 \% \text { l vs } 34 \% \text { C; } \\
\text { F:27\%l vs } 8 \% C \\
\text { Used financial } \\
\text { services from a bank: } \\
\text { M: } 49 \% \text { l vs } 32 \% C ; \\
\text { F:17\%l vs } 3 \% C \\
\text { Discussed financial } \\
\text { decisionmaking } \\
\text { M: } 56 \% \text { l vs } 23 \% C ; F: \\
75 \% \text { I vs } 21 \% C\end{array}$ & $\begin{array}{l}\text { Partner } \\
\text { communication in last } \\
\text { year } \\
\text { Self-esteem: } \\
\text { M: } 51 \% \text { l vs } 23 \% \text { C; F: } \\
70 \% \text { vs } 26 \% C \\
\text { Delay/avoid sex in last } \\
12 \text { months } \\
\text { F: } 76 \% \text { l vs } 66 \% \text { C } \\
\text { Sexuality } \\
\text { M: } 49 \% \text { l vs } 19 \% \text { C; F: } \\
67 \% \text { l vs } 26 \% \text { C } \\
\text { Contraception } \\
\text { M: } 49 \% \text { l vs } 19 \% \text { C; F: } \\
60 \% \text { l vs } 29 \% C \\
\text { Violence/Sexual Abuse } \\
\text { M: } 51 \% \text { l vs } 21 \% C \text {; F: } \\
62 \% \text { l vs } 24 \% C \\
\text { Condom use } \\
\text { M: } 56 \% \text { l vs21\%C; F: } \\
75 \% \text { l vs } 29 \% C\end{array}$ & $\begin{array}{l}\text { Among women: } \\
\text { Reported HIV testing: } \\
34 \% \text { baseline vs } 57 \% \text { post-I } \\
\text { Discussed avoiding STDs with } \\
\text { partner: } \\
91 \% \text { l vs } 89 \% \text { C } \\
\text { Discussed avoiding HIV with partner: } \\
87 \% \text { l vs } 77 \% \text { C }\end{array}$ & Not measured \\
\hline $\begin{array}{l}\text { SATZ }[31,37] \\
\text { CapeTown and Northern Province } \\
\text { NB: With regard to outcomes, } \\
\text { preliminary findings only are available. } \\
\text { Process evaluations have been } \\
\text { published[51,52]. }\end{array}$ & $\begin{array}{l}\text { Knowledge, } \\
\text { Attitudes, Social } \\
\text { Norms, Self-efficacy } \\
\text { were measured. } \\
\text { Knowledge } \\
\text { increased in } \\
\text { intervention schools }\end{array}$ & $\begin{array}{l}\text { No intervention } \\
\text { effects observed. } \\
\text { No effects on sub- } \\
\text { groups or for } \\
\text { secondary outcomes. }\end{array}$ & $\begin{array}{l}\text { Sexual Risk Behavior } \\
\text { Ever had sex: } 25.6 \% \text { l vs } 24.6 \% \text { C, } \\
\text { OR }=1.0 \text { [0.85-1.18] } \\
\text { Transition to sexual activity: } 19.0 \% \text { l } \\
\text { vs } 16.7 \% C \text {, OR }=1.05[0.85-1.29] \\
\text { Condon use at last sex: } 50.2 \% \text { l vs } \\
44.9 \% \text { C, OR }=1.13[0.79-1.62]\end{array}$ & Not measured \\
\hline
\end{tabular}




\section{Table 2: Study Results for Eight Youth HIV Prevention Interventions included in Systematic Review ${ }^{\mathbf{1}}$ (Continued)}

\begin{tabular}{|c|c|c|c|c|}
\hline $\begin{array}{l}\text { Stepping Stones }[11,38] \\
\text { Eastern Cape } \\
\text { NB: As knowledge, attitudes and } \\
\text { related measures were not reported in } \\
\text { main outcome paper, these categories } \\
\text { are not included here. } \\
\text { Results are from } 24 \text { month follow-up. }\end{array}$ & 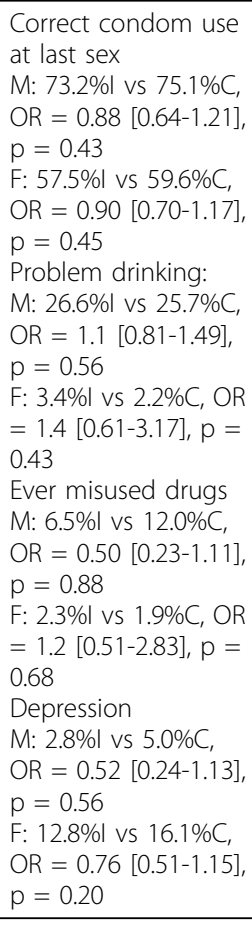 & \multicolumn{2}{|c|}{ 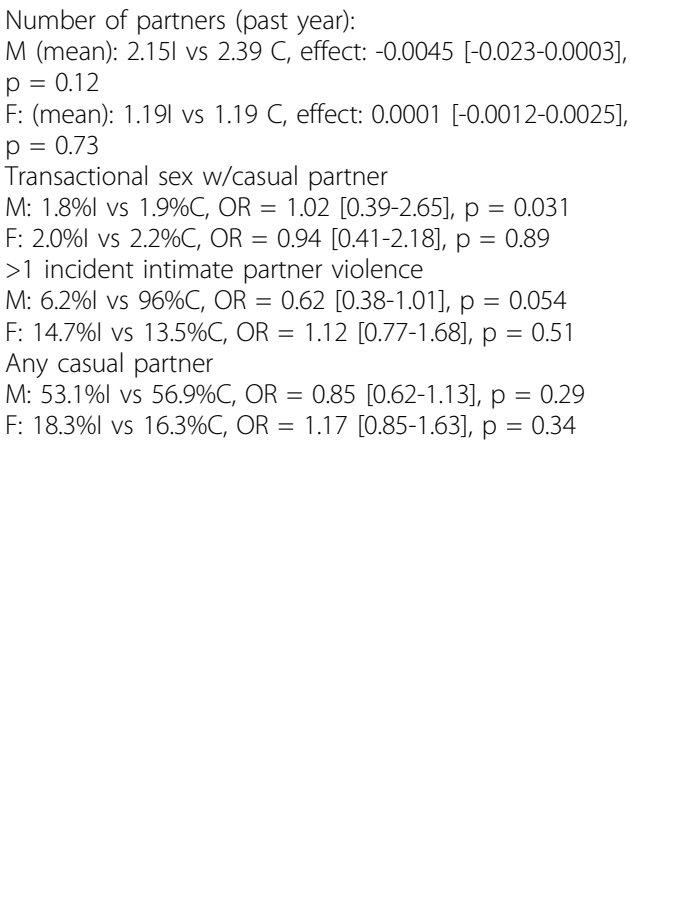 } & $\begin{array}{l}\text { Biological } \\
\text { HIV incidence: } \\
\text { F: } 5.651 \text { vs } 6.95 C \\
M: 1.4 I \text { vs } 1.29 C, \\
p=0.78 \\
\text { HSV-2: } \\
F: 5.351 \text { vs } 7.71 C \\
M: 1.461 \text { vs } 2.04 C, \\
p=0.36 \\
\text { Pregnancy } \\
F: 14.41 \text { vs } 11.6 C, \\
O R=1.45[0.92-2.28], \\
p=0.11 \\
M: 11.31 \text { vs } 12.9 C, \\
O R=0.88[0.60-1.31], \\
P=0.53\end{array}$ \\
\hline $\begin{array}{l}\text { Tshwane Peer Education and } \\
\text { Support Programme [36] } \\
\text { Tshwane (Pretoria), Gauteng } \\
\text { NB: In published results, measures of } \\
\text { significance were based on 'within } \\
\text { group' comparisons (eg., pre- and } \\
\text { post-test). For consistency, we present } \\
\text { 'between group' comparisons of the } \\
\text { post-test measures in I ( } \mathrm{n}=1572 \text { ) and } \\
\text { C ( } \mathrm{n}=532 \text { ) groups. }\end{array}$ & $\begin{array}{l}\text { Perception of peer } \\
\text { sexual activity } \\
\text { (most friends having } \\
\text { sex) } \\
20.7 \% \text { l vs } 25.4 \% \mathrm{C} \\
\text { Friends practice safe } \\
\text { sex } \\
51.0 \% \text { l vs } 54.2 \% \mathrm{C} \\
\text { Most friends drink } \\
\text { alcohol } \\
25.0 \% \text { l vs } 27.2 \% \mathrm{C}\end{array}$ & $\begin{array}{l}\text { Findings reported as } \\
\text { difference in scale } \\
\text { scores: } \\
\text { Psychological well- } \\
\text { being: } \\
52.441 \text { (SD6.8) vs } \\
51.51 C(S D 6.8) \\
\text { Personal control: } \\
7.481(\text { SD2.1) vs 8.10C } \\
\text { (SD2.1) } \\
\text { School Climate: } \\
57.491(\text { SD11.4) vs } \\
58.31 C(11.7)\end{array}$ & $\begin{array}{l}\text { Sexual experience: } \\
41.6 \% \text { l vs } 46.2 \% \text { C } \\
\text { Sex during past } 3 \text { months: } \\
36.9 \% \text { l vs } 30.8 \% \text { C } \\
\text { Multiple partners in past } 3 \text { months } \\
15.6 \% \text { lvs } 17.3 \% \text { C } \\
\text { Condom use every time in last } 3 \\
\text { months: } \\
59.3 \% \text { vs } 54.7 \% \text { C } \\
\text { Sex without consent } \\
\text { 19.7\%l vs } 20.8 \% \text { C } \\
\text { Current Alcohol Use } \\
21.9 \% \text { l vs } 22.7 \% \text { C } \\
\text { Excessive alcohol use } \\
13.1 \% 1 \text { vs } 18.0 \% \mathrm{C} \\
\text { Illicit drug use } \\
6.5 \% \text { l vs } 7.2 \% \mathrm{C}\end{array}$ & Not measured \\
\hline $\begin{array}{l}\text { IMAGE - Intervention with } \\
\text { Microfinance fir AIDS and Gender } \\
\text { Equity }[12,13]\end{array}$ & $\begin{array}{l}\text { Knowing HIV+ } \\
\text { person can look } \\
\text { healthy: } 91 \% \text { I vs } \\
87 \% \text { C, RR = } 1.09 \\
{[0.73-1.62]} \\
\text { Comfort discussing } \\
\text { sexuality in the } \\
\text { home: } 84 \% \text { l vs } 68 \% \\
\text { C, RR }=1.22 \text { [0.53- } \\
2.80]\end{array}$ & $\begin{array}{l}\text { Communication in } \\
\text { h'hold about sex (past } \\
\text { year): } 74 \% \text { l vs } 50 \% \text { C, } \\
\text { RR }=1.46[1.01-2.12]\end{array}$ & $\begin{array}{l}\text { >1 sexual partner in past year: } \\
4 \% \text { l vs } 3 \% \text { C, RR }=0.95[0.40-2.27] \\
\text { Unprotected last sex, non-spousal } \\
\text { partner: } 55 \% \text { l vs } 78 \% \text { C, RR }=0.76 \\
\text { [0.60-0.96] } \\
\text { ?HIV test: } 29 \% \text { l vs } 18 \% \text { C, RR }=1.64 \\
\text { [1.06-2.56] }\end{array}$ & $\begin{array}{l}\text { No biological } \\
\text { outcome measures } \\
\text { for analysis of } \\
\text { women under age } \\
35\end{array}$ \\
\hline
\end{tabular}

${ }^{1}$ All data are presented for combined male and female results, except where published results included only gender-disaggregated results (eg., Stepping Stones and Adolescent Livelihoods projects). ${ }^{2}$ Indicates significant findings for sub-sample of males in Intervention vs Control at followup (not shown). ${ }^{3}$ Indicates significant findings for sub-sample of females in Intervention vs Control at followup (not shown). 
Some interventions incorporated multi-level approaches, as in the two curricula in HAPS addressing sexual risk reduction and alcohol [32]. Two other projects [34,35] addressed multiple, discrete topics within one intervention, such as HIV prevention and financial skills [35], HIV and pregnancy prevention, or 'dual protection' [33], sexual and alcohol risk reduction coupled with positive leisure time use [34], or school-level factors [36]. All interventions, both school- and group-based, used written curricula, except for the TPE Project, in which facilitators and peer educators developed role plays and presentations.

\section{Intervention Development and Cultural Adaptation}

Considerable variation existed in the cultural adaptation of interventions to South Africa. Five curricula were developed in the US or UK, while Stepping Stones was developed in Uganda. These were adapted via qualitative research [31,32] or participatory action research $[11,13,33,34]$ with the target audience. One of the five adapted specific modules from several effective interventions in the US and South Africa [33], rather than entire curricula $[11,31,32,34]$. Two interventions were developed locally $[35,36]$, with one not using a formal curriculum [36].

\section{Theoretical Framework}

Most studies were based on social cognitive models of behavioural change [31-34,36], although possible limitations of these individual risk-reduction models for nonWestern contexts were acknowledged [31,33] (Additional file 1, Table S3). To address this, some studies expanded existing theoretical frameworks to include socio-cultural factors [31], or used individual and grouplevel theories together [33]. Three studies used an empowerment model based on the theories of Paolo Freire $[11,13,33]$.

\section{Thematic Focus and HIV Causal Pathways}

Each intervention had a unique thematic focus within overall HIV prevention goals (Table 1), reflecting the hypothesised causal pathways to HIV infection. Generally, the causal pathways represented probable influences on HIV infection and associated risk factors. Stepping Stones and IMAGE, for example, addressed genderbased violence as an important influence on HIV risk, with reduction of intimate partner violence a key intervention message [11]. HAPS and HealthWise both strongly emphasized alcohol use as a risk factor for HIV infection [32,34]. Along with Stepping Stones and IMAGE, Mpondombili focused on gender equity, adopting an empowerment approach to challenge negative gender values [33]. Adolescent Livelihoods and IMAGE focused on the economic context of HIV risk, specifically the attainment of vocational or financial life skills aimed at individual empowerment $[13,35]$. In addition to peer social norms related to HIV prevention, the TPE Project [36] addressed school-level factors that could support students' HIV prevention goals, while HealthWise focused on positive leisure time use [34]. While these are not, with the exception of IMAGE, truly 'structural interventions', they are focused on key structural factors that affect HIV risk at the individual level, as well as their social context.

\section{Intervention Delivery}

Five interventions used standard, classroom-based methods [31-34,36]. Some studies used schools in innovative ways, including Healthwise's after-school activities [34], or as a meeting place for in-school and out-of-school youth in Adolescent Livelihoods [35], and for extra-curricular sessions in Stepping Stones [11]. The main differences, however, between the school-based and nonschool-based approaches were the individual versus group approaches. Importantly, the use of group-based intervention delivery appears to have fostered positive shifts in group norms regarding HIV risk behaviours $[11,13,35]$.

\section{Who Delivered the Intervention?}

In 4 of the 5 school-based interventions [31-34], teachers had a main role in delivering the intervention. The SATZ and Mpondombili projects trained peer educators to co-facilitate the intervention with teachers in classrooms [31,33]. The TPE Project relied on peer educators with guidance from post-graduate student advisors, and the support, but not active involvement, of teachers [36]. Teachers generally were responsible for overseeing peer educators as well as the curriculum, with assistance from an intervention team [31-33].

Peer educators of the same age or slightly older participated in all but one school-based intervention [31-33,36]. Several interventions had older peer facilitators, who functioned as mentors and were responsible for leading structured group discussions $[11,35]$. Usually, peer educators were selected via a competitive process $[9,33]$, or via nomination by student peers $[32,36]$. In some projects, such as TPE, teachers made the final selection [36]. Training generally consisted of strengthening the peer educators' knowledge base, and developing facilitation, interpersonal and public speaking skills. Length of training varied, however, from a 1-2 day workshop in the Tshwane and HAPS projects, to six months in Mpondombili. In HAPS, peer leaders led discussions following audiotaped vignettes about teenagers' dilemmas regarding alcohol use and sexual activity [32]. In Mpondombili, as well as HAPS, SATZ and HealthWise, teachers also received extensive training, ranging 
from 2-3 days to several multi-day workshops (Additional file 1, Table S3). Overall, peer educators were popular with students, but required high levels of training and support for their expected roles.

\section{Intervention Duration, Intensity and Reinforcement of Key Messages}

The eight interventions varied greatly in number of sessions, programme duration, and frequency of sessions (Additional file 1, Table S3), ranging from 8 weeks to six months, with two interventions providing booster sessions [31,34]. The number of intervention modules ranged from 10-16, although there was a wider range in the number or sessions, as some modules took 2-3 sessions to complete [32,34]. In-school and out-of-school interventions were of similar duration.

\section{Summary of Review Findings}

In summary, the review finds that, although within these trials the effects of most interventions on reported sexual risk behaviour or biological outcomes were limited, common elements related to their impact on secondary outcomes can be discerned, as well as aspects of intervention delivery. These include: 1) a focus on at least one social/structural risk factor, as in the emphasis on gender, poverty and alcohol in these eight interventions, 2) using group-based delivery to change social norms, 3) within schools, demonstrating the need to use additional personnel, perhaps from outside the school setting, to deliver interventions, thus relieving a burden on teachers, and 4) directing intervention efforts at the school, as well as individual, level. In addition, several studies demonstrate the potential of structural interventions to bring about changes in HIV-related risk behaviours.

\section{Discussion}

How do the findings from this review answer the question 'what works' to prevent HIV infection in young people in southern Africa? Importantly, the review identifies key elements associated with intervention impact, and highlights promising approaches in youth HIV prevention for South Africa and similar settings. These eight interventions, most of them rigorously designed with regard to intervention and evaluation, indicate progress toward improved HIV prevention, based on behavioural proxy measures for HIV infection. However, given the range of interventions that have been tested, differences in the relative strengths and weaknesses of evaluation designs, and the limited effectiveness of many interventions, a definitive assessment of 'what works' is not possible. Yet important lessons are learned, leading to specific recommendations for future research.

What are the lessons learned from the eight studies included in this review? First, moving beyond individual-level measures of knowledge and psychosocial factors to address social and structural factors underlying HIV risk is the main success of these interventions. An important feature - and their area of greatest impact - was the focus on HIV causal pathways relevant to southern Africa, namely gender, sexual coercion, alcohol use and economic risk. Results of the two other major youth intervention trials in sub-Saharan Africa, MkV and RDS, support the importance of addressing social and institutional, as well as individual level, factors to change population-level norms about HIV risk behaviours $[9,10,21]$. However, addressing social factors is not the same as implementing a 'structural intervention', as in the IMAGE project where women's access to economic resources apparently increased personal empowerment, leading to reduced sexual risk behaviour [42], a finding observed in the larger trial as well as the sub-group of younger women. Similarly, Stepping Stones led to changes in gender beliefs and values, thus impacting the structural context of risk [11]. An important second lesson, then, is the need for interventions to adopt structural approaches that can alter the context of young people's HIV risk. Although structural interventions are often critiqued as 'social development' rather than focused health interventions [14], in fact these studies offer several important examples of how targeted structural approaches can change individual behaviour. The HealthWise intervention offered young people alternatives for leisure time use, while in the Adolescent Livelihoods project young people learned life skills, such as numeracy, that may enhance vocational and educational success. Promising economic interventions are also being tested in other sub-Saharan African settings [43]. Third, changing social norms related to HIV risk and protective behaviours is important. Stepping Stones' success was clearly associated with altering beliefs about gender and HIV risk, particularly among men, and with offering viable alternative normative behaviours $[11,44]$. One way that group intervention approaches, such as IMAGE and Stepping Stones, generate positive social norms is through engaging participants in collective critical thinking, thereby fostering self-esteem and individual empowerment [45]. Community mobilisation, employed effectively among women in the IMAGE project and in other promising interventions $[13,14,45]$, is also an important component. Importantly, most school-based interventions do not use a group approach, but are delivered didactically by teachers in classrooms, relying on the ability of students to act individually on information received. A simple way to address this would be for school-based interventions to include more group-based, rather than didactic, learning. A fourth lesson learned is thus the need to engage schools differently in HIV prevention, including use of personnel 
other than teachers to deliver interventions. While peer education is popular among students, this review supports other findings that offer little evidence for its ability to increase intervention impact $[46,47]$. One approach with certain advantages over teachers, who often resist teaching sexuality education, and same-age peers, who may sometimes have difficulty commanding the necessary authority to run a classroom $[41,47]$, would be to use older youth as 'mentors'. School mentors could work in partnership with teachers who request to teach sexuality education and HIV prevention, but would relieve the reliance on teachers who do not want this responsibility.

Of the five school-based interventions reviewed, four experienced serious implementation problems, leading to calls for interventions to target the school level as well as individual students [31,32,34]. Interventions in South African schools experience challenges to fidelity, due to scheduling disruptions, high levels of student and teacher absenteeism, frequent violence and other school-level issues [48]. These findings thus suggest the need to change how school interventions are implemented, with enormous potential to improve on current models. Yet in spite of the difficulties, schools have some important advantages for intervention delivery, including the ability to reach a large number of youth $[49,50]$, suggesting that working to improve schoolbased intervention models is an important priority. Methods from the education sector could inform HIV prevention interventions, including the use of school mentors, teacher training in participatory learning approaches, and efforts at school-level change [48]. At the same time, recognition of the considerable variation in youth needs is important, and interventions in community or other venues designed to reach out-of-school youth, or older adolescents, are also research priorities.

\section{Conclusions}

Based on the findings from this review - and from the $\mathrm{MkV}$ and RDS trials - specific recommendations for a 'third generation' of youth HIV prevention interventions can be made. These include 1) conducting trials of youth-focused structural interventions, with the aim of altering the structural context of HIV risk; 2) developing new approaches for schools, including interventions that target school-level factors and engage schools as active partners, including mobilising the broader 'school community' of students, teachers, parents and community members; and 3) ensuring that future trials have better measurement and more rigorous designs, including HIV incidence - or another comparable biological measure, such as HSV-2 - as the primary outcome, as well as longer-term follow-up. These recommendations can help to address remaining gaps in knowledge about youth HIV prevention, in spite of important lessons learned from the 'second generation' interventions reviewed here. These include a more definitive understanding of the merits of multi- versus single-component interventions, or of broad-based versus more narrowly targeted approaches, and developmental considerations, like age, gender, sexual activity, and schooling status. These recommendations can help to improve youth HIV prevention research, where - in spite of great progress - no interventions have yet demonstrated a reduction in HIV incidence. This remains the most important - and essential - marker of intervention success.

Additional file 1: Table S3. Intervention Design, Content and

Characteristics for Eight Youth HIV Prevention Interventions included in Systematic Review

Click here for file

[http://www.biomedcentral.com/content/supplementary/1471-2458-10102-S1.DOC ]

\section{Acknowledgements}

The Africa Centre for Health and Population Studies is funded by the Wellcome Trust, UK for core support and surveillance [082384/Z/07/Z].

\section{Author details}

'Brown University, Population Studies and Training Center, and Department of Medicine, Warren Alpert School of Medicine, Providence, RI USA. ${ }^{2}$ Africa Centre for Health and Population Studies, University of KwaZulu Natal, Somkhele, South Africa. ${ }^{3}$ Centre for Paediatric Epidemiology, Institute of Child Health, University College London, London, UK. ${ }^{4}$ National Centre in HIV Social Research, University of New South Wales, Sydney, Australia.

\section{Authors' contributions}

$\mathrm{AH}$ and MLN designed the review, and contributed to all phases of the research and writing. In addition, AH collected and analyzed the data for each study included in the review. JI contributed to analysis and interpretation of results, as well as preparation of the manuscript. GH contributed to data analysis and preparation of the manuscript. All authors reviewed and approved the final version of this manuscript.

\section{Competing interests}

The authors declare that they have no competing interests.

Received: 14 September 2009 Accepted: 26 February 2010 Published: 26 February 2010

\section{References}

1. UNAIDS: AIDS Epidemic. Update. Joint United Nations Programme on HIV/ AIDS. Geneva, Switzerland 2008. [http://data.unaids.org/pub/Report/2009/ JC1700_Epi_Update_2009_en.pdf], Accessed 8 March 2010.

2. Ross D, Dick B, Ferguson J: Preventing HIV/AIDS in Young People: A Systematic review of the evidence from developing countries. Report of the UNAIDS Inter-agency Task Team on Young People World Health Organization Technical Report Series 9382006

3. Laga M, Schwärtlander B, Pisani E, Sow PS, Caraël M: To stem HIV in Africa, prevent transmission to young women. AIDS 2001, 15:931-934.

4. Stirling $M$ Rees $H$, Kasedde $S$, Hankins $C$ : Introduction: addressing the vulnerability of young women and girls to stop the HIV epidemic in southern Africa. AIDS 2008, 22:S1-S3.

5. Kirby D, Laris BA, Rolleri LA: Sex and HIV Education Programs: Their Impact on Sexual Behaviors of Young People throughout the World. J Adolesc Health 2007, 40:206-17. 
6. Wellings K, Collumbien M, Slaymaker E, Singh S, Hodges Z, Patel D, Bajos N: Sexual behaviour in context: a global perspective. Lancet 2006, 368(9548):1706-28.

7. Wegbreit J, Bertozzi S, DeMaria LM, Padian NS: Effectiveness of HIV prevention strategies in resource-poor countries: tailoring the intervention to the context. AIDS 2006, 20(9):1217-35.

8. Kinsman J, Nakiyingi J, Kamali A, Carpenter L, Quigley M, Pool R, Whitworth J: Evaluation of a comprehensive school-based AIDS education programme in rural Masaka, Uganda. Health Educ Rsch 2001, 16(1):85-100.

9. Ross DA, Changalucha J, Obasi AIN, Todd J, Plummer M, CleophasMazige B, Anemona A, Everett D, Weiss HA, Mabey DC, Grosskurth H, Hayes RJ: Biological and behavioural impact of an adolescent sexual health intervention in Tanzania: a community-randomized trial. AIDS 2007, 21:1943-1955.

10. Cowan FM: Cluster randomized trail of a multi-component HIV prevention intervention for young people in rural Zimbabwe. University College London, Technical Briefing Note 2008.

11. Jewkes R, Nduna M, Levin J, Jama N, Dunkle K, Puren A, Duvvury N: Impact of stepping stones on incidence of HIV and HSV-2 and sexual behaviour in rural South Africa: cluster randomised controlled trial. British Med Journal 2008, 337:a506, doi: 10.1136/bmj.a506.

12. Pronyk P, Hargreaves J, Kim JC, Morison LA, Phetla G, Watts C, Busza J, Porter JD: Effect of a structural intervention for the prevention of intimate-partner violence and HIV in rural South Africa: a cluster randomised trial. Lancet 2006, 368:1973-83.

13. Pronyk PM, Kim JC, Abramsky T, Phetla G, Hargreaves JR, Morison LA, Watts C, Busza J, Porter JD: Combined microfinance and training intervention can reduce HIV risk behaviour in young female participants. AIDS 2008, 22:1659-66.

14. Rao Gupta G, Parkhurst JO, Ogden JA, Aggleton P, Mahal A: Structural approaches to HIV prevention. Lancet 2008, 372:764-5.

15. Buve A, Newell ML, eds: Vulnerability of Young Women and Girls to HIV in sub-Saharan Africa. AIDS 2008, 22(Supplement 4):S1-S86.

16. Gallant M, Maticka-Tyndale E: School-based HIV prevention programmes for African youth. Soc Sci Med 2004, 58(7):1337-51.

17. Paul-Ebhohimhen VA, Poobalan A, van Teijlingen ER: A systematic review of school-based sexual health interventions to prevent STI/HIV in subSaharan Africa. BMC Public Health 2008, 8:4

18. Oakley A, Fullerton D, Holland J, Arnold $S$, et al: Sexual health education interventions for young people: a methodological review. British Med Journal 1995, 310:158-62.

19. Stanton B, Kim N, Galbraith J, Parrott M: Design Issues Addressed in Published Evaluations of Adolescent HIV-Risk Reduction Interventions: A Review. Journal of Adolescent Health 1996, 18:387-396.

20. Mema kwa Vijana: Rethinking how to prevent HIV in young People: Evidence from two large randomized controlled trials in Tanzania and Zimbabwe. Policy Briefing Paper No. 102008

21. Cowan FM, Pettifor A: HIV in adolescents in sub-Saharan Africa. Curr Opin HIV AIDS 2009, 4(4):288-93.

22. UNAIDS: Knowing your Epidemic, 2007 Annual Report. Geneva: UNAIDS 2008http://data.unaids.org/pub/Report/2008/jc1535_annual_report07_en. pdf, 8 February 2010.

23. Ross DA, Wight D, Dowsett G, Buve A, Obasi AIN: The weight of evidence: a method for assessing the strength of evidence on the effectiveness of HIV prevention interventions among young people. Preventing HIV/AIDS in Young People: A Systematic review of the evidence from developing countries. Report of the UNAIDS Inter-agency Task Team on Young People World Health Organization Technical Report Series 938Ross D, Dick B, Ferguson J 2006, Chapter 4.

24. Pettifor AE, Kleinschmidt I, Levin J, Rees HV, MacPhail C, MadikizelaHlongwa L, Vermaak K, Napier G, Stevens W, Padian NS: A communitybased study to examine the effect of a youth HIV prevention intervention on young people aged 15-24 in South Africa: results of the baseline survey. Tropical Medicine and International Health 2005, 10(10):971-80

25. Goldstein S, Usdin S, Scheepers E, Japhet G: Communicating HIV and AIDS, what works? A report on the impact evaluation of Soul City's fourth series. J Health Comm 2005, 10(5):465-83.

26. Visser MJ, Schoeman JB, Perold JJ: Evaluation of HIV/AIDS prevention in South African schools. J Health Psychology 2004, 9(2):263-280.
27. Magnani R, Macintyre K, Karim AM, Brown L, Hutchinson P, Kaufman C: The impact of life skills education on adolescent sexual risk behaviors in KwaZulu/Natal, South Africa. J Adol Health 2005, 36(4):289-304.

28. James S, Reddy P, Ruiter RA, McCauley A, Borne van den B: Impact of an HIV and AIDS Life Skills Program on Secondary School Students in KwaZulu/Natal, South Africa. AIDS Ed Prev 2006, 18(4):281-94.

29. James S, Reddy P, Ruiter RA, Taylor M, Jinabhai CC, Van Empelen P, Borne Van den B: The effects of a systematically developed photonovella on knowledge, attitudes, communication and behavioural intentions with respect to sexually transmitted infections among secondary school learners in South Africa. Health Promotion International 2005, 20(2):157-65

30. Petersen I, Mason A, Bhana A, Bell CC, McKay M: Mediating social representations using a cartoon narrative in the context of HIV/AIDS: the Ama Qhawe Family Project in South Africa. Journal of Health Psychology 2006, 11(2):197-208.

31. Mathews C: Presentation, SATZ and HealthWise Interventions. Experts Workshop on 'Youth School-based Intervention Strategies'. Africa Centre for Health and Population Studies, Mtubatuba, South Africa, October 29-31, 2007.

32. Karnell A, Cupp PK, Zimmerman RS, Feist-Price S, Bennie T: Efficacy of an American Alcohol and HIV Prevention Curriculum adapted for Use in South Africa: Results of a Pilot Study in Five Township Schools. AIDS Education and Prevention 2006, 18(4):295-310.

33. Exner T, Harrison A, Hoffman S, Smit J, Mantell J, Nzama T, Stein Z: Schoolbased Intervention reduces Sexual Risk and Changes Gender Role Norms: The Mpondombili Project in Rural South Africa. XVIth International AIDS Conference, Toronto, Canada, August 13-18th, 2006.

34. Smith EA, Palen LA, Caldwell LL, Flisher AJ, Graham JW, Mathews C, Wegner $L$, Vergnani $T$ : Substance use and sexual risk prevention in CapeTown, South Africa: an evaluation of the HealthWise program. Prev Sci 2008, 9(4):311-21.

35. Hallman K, Govender K, Roca E, Pattman R, Mbatha E, Bhana D: Enhancing financial literacy, HIV/AIDS skills, and safe social spaces among vulnerable South African youth. New York: Population Council, Transitions to Adulthood Brief No.4 2007

36. Visser MJ: HIV/AIDS prevention through peer education and support in secondary schools in South Africa. SAHARA J 2007, 4(3):678-94.

37. Aaro LE, Flisher A, Kaaya S, Onya H, Fuglesang M, Klepp Kl, Schaalma H: Promoting sexual and reproductive health in early adolescence in South Africa and Tanzania: Development of a theory and evidence-based intervention program. Scandinavian Journal of Public Health 2006 34:150-158.

38. Jewkes R, Nduna M, Levin J, Jama N, Dunkle K, Khuzwayo N, Khuzwayo N, Koss M, Puren A, Wood K, Duvvury N: A cluster randomized-controlled trial to determine the effectiveness of Stepping Stones in preventing HIV infection and promoting safer sexual behaviour amongst youth in the rural Eastern Cape, South Africa: trial design, methods and baseline findings. Trop Med Int Health 2006, 11(1):3-16.

39. Cowan FM, Pascoe SJ, Langhaug LF, Dirawo J, Chidiya S, Jaffar S, Mbizvo M, Stephenson JM, Johnson AM, Power RM, Woelk G, Hayes RJ: The Regai Dzive Shiri Project: a cluster randomised controlled trial to determine the effectiveness of a multi-component community-based HIV prevention intervention for rural youth in Zimbabwe-study design and baseline results. Tropical Medicine and International Health 2008 , 13(10):1235-44.

40. Wegner L, Flisher AJ, Caldwell LL, Vergnani T, Smith EA: Healthwise South Africa: cultural adaptation of a school-based risk prevention programme. Health Educ Res 2008, 23(6):1085-96.

41. Mantell J, Harrison A, Hoffman S, Smit JA, Stein ZA, Exner TM: The Mpondombili Project: Preventing HIV/AIDS and Unintended Pregnancy among Rural South African School-going Youth. Repro Health Matters 2006, 14(28):113-122.

42. Kim JC, Pronyk PM, Barnett T, Watts C: Exploring the role of economic empowerment in HIV prevention. AIDS 2008, 22(Suppl 4):S57-S71.

43. Duflo E, Dupas $P$, Kremer M, Sinei S: Education and HIV/AIDS Prevention: Evidence from a Randomized Evaluation in Western Kenya. 2006. [http:// www.povertyactionlab.org]

44. Hayes RJ: Prevention of HIV in young people in Africa. British Med Journal 2008, 337:a743.

45. Friedman SR, O'Reilly KR: Sociocultural interventions at the community level. AIDS 1997, 11(suppl A):s201-208. 
46. Ross DA: Approaches to Sex Education: Peer-Led or Teacher-Led? PLOS Med 2008, 5(11):e229.

47. Kim CR, Free C: Recent evaluations of the peer-led approach in adolescent sexual health education: a systematic review. Perspectives Sexual Repro Health 2008, 40(3):144-51.

48. Jansen J: Bodies Count: AIDS Review 2006 Pretoria, South Africa: Centre for the Study of AIDS, University of PretoriaCrewe M 2006

49. Lloyd C: The Role of Schools in Promoting Sexual and Reproductive Health among Adolescents in Developing Countries New York: Population Council Working Paper No. 62007.

50. Plummer ML, Wight D, Wamoyi J, Nyalali K, Ingall T, Mshana G, Shigongo ZS, Obasi Al, Ross DA: Are schools a good setting for adolescent sexual health promotion in rural Africa? A qualitative assessment from Tanzania. Health Educ Research 2007, 22(4):483-499,

51. Namisi FS, Flisher AJ, Overland S, Bastien S, Onya H, Kaaya S, Aarø LE: Sociodemographic variations in communication on sexuality and HIV/ AIDS with parents, family members and teachers among in-school adolescents: A multi-site study in Tanzania and South Africa. Scand J Public Hlth 2009, 37(Suppl 2):65-74.

52. Mukoma W, Flisher AJ, Ahmed N, Jansen S, Mathews C, Klepp KI, Schaalma H: Process evaluation of a school-based HIV/AIDS intervention in South Africa. Scand J Public Hlth 2009, 37(Suppl 2):37-47.

\section{Pre-publication history}

The pre-publication history for this paper can be accessed here: http://www. biomedcentral.com/1471-2458/10/102/prepub

doi:10.1186/1471-2458-10-102

Cite this article as: Harrison et al:: HIV prevention for South African youth: which interventions work? A systematic review of current evidence. BMC Public Health 2010 10:102.

\section{Submit your next manuscript to BioMed Central and take full advantage of:}

- Convenient online submission

- Thorough peer review

- No space constraints or color figure charges

- Immediate publication on acceptance

- Inclusion in PubMed, CAS, Scopus and Google Scholar

- Research which is freely available for redistribution

Submit your manuscript at www.biomedcentral.com/submit 